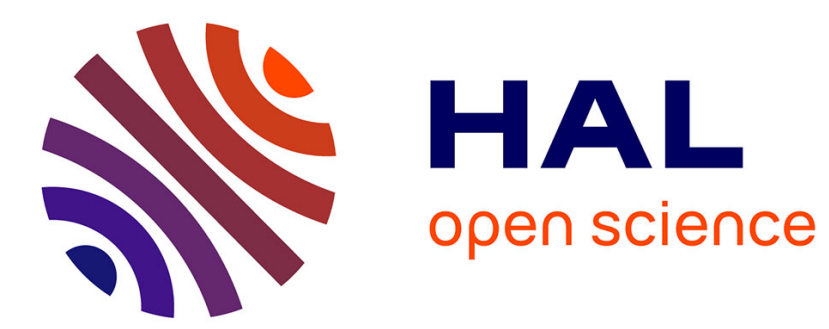

\title{
Propagative modes of plastic deformation
}

P. Hähner, M. Zaiser

\section{To cite this version:}

P. Hähner, M. Zaiser. Propagative modes of plastic deformation. Journal de Physique IV Proceedings, 1993, 03 (C7), pp.C7-1995-C7-2004. 10.1051/jp4:19937319 . jpa-00251964

\section{HAL Id: jpa-00251964 https://hal.science/jpa-00251964}

Submitted on 1 Jan 1993

HAL is a multi-disciplinary open access archive for the deposit and dissemination of scientific research documents, whether they are published or not. The documents may come from teaching and research institutions in France or abroad, or from public or private research centers.
L'archive ouverte pluridisciplinaire HAL, est destinée au dépôt et à la diffusion de documents scientifiques de niveau recherche, publiés ou non, émanant des établissements d'enseignement et de recherche français ou étrangers, des laboratoires publics ou privés. 


\title{
Propagative modes of plastic deformation
}

\author{
P. HÄHNER ${ }^{(1)}$ and M. ZAISER
}

Max-Planck-Institut für Metallforschung, Institut für Physik, P.O. Box 800 665, 70506 Stuttgart, Germany

\begin{abstract}
A major objective of the theory of defects is to relate the mechanical behaviour of macroscopic materials to the spatio-temporal evolution of the microstructure. The present paper deals with the dislocation-dynamical foundations of plastic instabilities and the propagation of slip in coherent plastic deformation modes (solitary plastic waves). Solitary waves arise from a proper balance between nonlinear localization effects and dispersion. By the dislocation dynamical approach, both nonlinear interactions and spatio-temporal couplings are accessible in a quantitative way. Particularly, intrinsic length scales may be identified, in order to address the problem of propagation velocity selection. This is illustrated by means of various models of propagative plastic instabilities observed in tensile tests. The model assumptions are as follows: 1.) The microscopic cause of the repeated non-uniform yielding of the Portevin-Le Chatelier (PLC) effect is dynamic strain-ageing, while the macroscopic propagation of PLC bands is controlled by intergranular incompatibility stresses. 2.) Lüders bands in polycrystals result from a dislocation dynamics which is diffusion-like (owing to the random grain orientation) and bistable (owing to the stabilizing effect of the grain boundaries). 3.) This is to be compared with Lüders bands in single crystals where the dislocation-poor initial state is unstable and band propagation is traced back to an interplay of cross-slip and non-axial stresses. 4.) Thermomechanical fronts arise from the interplay between heat generation during plastic deformation, heat conduction, and strain hardening.
\end{abstract}

\section{Introduction}

The present paper gives an overview of recent progress in the modelling of phenomena associated with the nucleation and propagation of localized modes of plastic deformation in a tensile test. In view of the variety of the observed phenomena, the paper focuses on those aspects that proved to be important in solving long-standing problems related to the propagation of plastic waves. Particular emphasis is laid on the question which physical mechanisms govern the spatio-temporal dynamics of the different types of propagative deformation modes. The results presented may stimulate further experimental investigations in order to check the theoretical findings.

For modelling to be more than a way of trial, a couple of questions have to be addressed. It has proved to be useful to start from the idea that localized modes of plastic deformation belong to the more general notion of plastic instabilities a classification of which was given by Estrin and Kubin [1] in terms of linear stability analysis (LSA). In the present paper we shall deal with instabilities associated with a negative strain-rate sensitivity $S$ (type $S$ ), a negative strain-hardening coefficient $h$ (type $h$ ), and with heat production during plastic deformation (type $T$ ).

However, each particular type of propagative instability is determined by a complex ensemble of interrelated factors (Fig. 1) in which instability of uniform plastic flow is just one building block. It is necessary to investigate quantitatively the length and time scales involved in order to identify those 


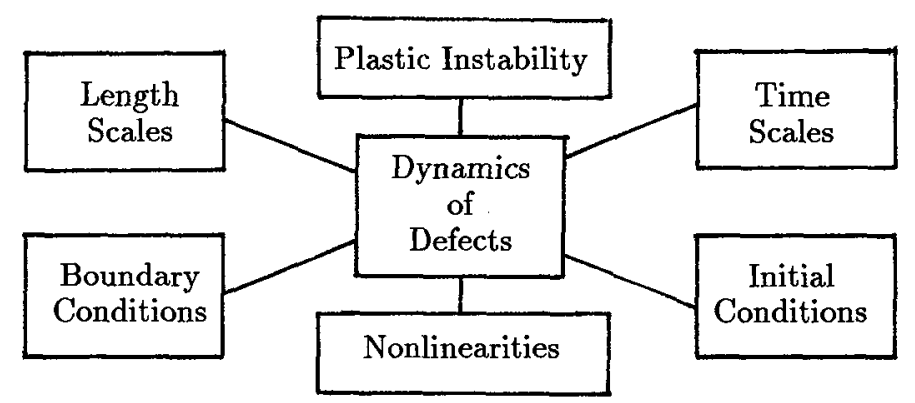

Figure 1: A collection of aspects that are important in the modelling of propagative instabilities.

spatial couplings and rate-controlling processes, respectively, that govern the overall propagation behaviour. Depending on the material and deformation conditions this consideration may reveal a diffusion-like dislocation dynamics, either due to the random grain orientation in a polycrystal or due to cross-slip (Sect. 3.1), or the spreading of the deformation mode may be controlled by second order stress gradients that arise either due to incompatibilities of plastic strain (Sect. 2) or due to a non-axiality of the stress state (Sect. 3.1), or by heat conduction (Sect. 4).

Even for the comparatively simple geometry of a uniaxial tensile test boundary conditions may play an important rôle. This will be illustrated with a model of the Portevin-Le Châtelier (PLC) effect, the solution of which depends on whether repeated yielding is assigned to a periodic travelling wave train in an infinitely long tensile specimen or whether zero plastic strain rate at the specimen grips is taken into account (Sect. 2). Initial conditions are important inasmuch as the aforementioned phenomena are preceded by some kind of nucleation process. Depending on the localization of the nucleus, either a propagating plastic wave or uniform flow may develop. This is an important aspect that is beyond the scope of LSA. Moreover, LSA is inadequate with regard to the fact that the actual appearance of a propagative instability depends on the nonlinearities involved in the deformation process. In this context, we recall the well-known fact that solitary wave propagation results from a proper balance of nonlinear localization and dispersion.

The dynamics of defects (dislocations and, if necessary, point defects) does not only give access to the aforementioned aspects of propagative instabilities, but, in most cases, it is necessary to proceed from dislocation densities as variables of state in such a manner as to obtain a complete formulation of the deformation path. As the case may be, the number of structure variables may be reduced using an adiabatic elimination of the defect densities evolving fastest. It is clear from what has been stated above that the dynamics is, in general, constituted by a set of coupled nonlinear partial differential equations relating the microscopic structure to the macroscopic deformation state. In what follows this is illustrated by means of the PLC effect (Sect. 2), Lüders band propagation both in single and in polycystals (Sect. 3), and thermomechanical fronts emerging during low-temperature deformation (Sect. 4). It is pointed out which processes seem to be decisive in each case and what type of information is obtained from theory. Only the most important results are quoted while the reader interested in more details is referred to the literature.

\section{The Portevin-Le Châtelier Effect}

It is well-established that the repeated yielding associated with the PLC effect arises from a collective dislocation behaviour in dynamic strain ageing (DSA) alloys [2]. In a certain range of plastic strain rates $\dot{\epsilon}$ and temperature $T$, DSA may lead to to a negative strain-rate sensitivity of the flow stress $\sigma_{\mathrm{f}}, S=\partial \sigma_{\mathrm{f}} / \partial \ln \dot{\epsilon}<0$, and, hence, to an instability of plastic flow (type $S$ instability [1]). With regard to this negative characteristic, the PLC effect is in close analogy to the Gunn effect (negative differential electron mobility) [3]. This is illustrated in Table 1. Thus, the mathematical formulation 
Table 1: Comparison between the PLC and the Gunn Effect ( $n$ and $D$ are the density and the diffusion coefficient of electrons, respectively).

\begin{tabular}{|c||c|}
\hline GUNN EFFECT & PLC EFFECT \\
\hline \multicolumn{2}{|c||}{ generalized fluxes: } \\
\hline electron drift velocity $v_{\mathrm{e}}$ & flow stress $\sigma_{\mathfrak{f}}$ \\
\hline \multicolumn{2}{|c|}{ generalized forces: } \\
\hline electric field $E$ & plastic strain rate $\dot{\epsilon}$ \\
\hline \multicolumn{2}{|c|}{ further analogies: } \\
\hline $\begin{array}{c}\text { electron flux } j \\
\text { electric potential } U \\
\text { diffusive flux }-D \partial_{x} n\end{array}$ & $\begin{array}{c}\text { external stress } \sigma_{\text {ext }} \\
\text { cross-head velocity } \dot{L} \\
\text { internal stress } \sigma_{\text {int }}\end{array}$ \\
\hline charge conservation: $\partial_{t} n+\partial_{x} j=0$ & cylindrical specimen: $\partial_{x} \sigma_{\text {ext }}=0$ \\
\hline$E$ irrotational: $U=-\int E \mathrm{~d} x$ & machine condition: $L=\int \dot{\epsilon}$ d $x+M_{\text {eff }} l \partial_{t} \sigma_{\text {ext }}$ \\
\hline superposition of fluxes: $j=n v_{\mathrm{e}}-D \partial_{x} n$ & superposition of stresses: $\sigma_{\text {ext }}=\sigma_{\mathrm{f}}-\sigma_{\text {int }}$ \\
\hline Poisson equation: $\partial_{x} E=-4 \pi n$ & internal stress: $\sigma_{\text {int }}(c f$. text $)$ \\
\hline
\end{tabular}

of the Gunn effect [4] may serve as a guideline in the present case. In both cases there are, in principle, two possible testing conditions depending on whether the generalized fluxes or forces are controlled: in the first case (stress control) the PLC effect manifests itself in staircase-like stress-strain curves whereas in the second case (strain rate control) different types of serrated stress-strain curves labelled as types $\mathrm{A}, \mathrm{B}$ and $\mathrm{C}$ are observed.

A useful model of the PLC effect that was first presented by Penning [5] and investigated further by Kubin and Estrin [2] is based on a decomposition of the flow stress into a strain hardening contribution and a rate-sensitive friction stress $f$,

$$
\sigma_{\mathrm{f}}\left(\epsilon_{0}, \dot{\epsilon}_{0}\right)=\sigma_{0}+h \epsilon_{0}+f\left(\dot{\epsilon}_{0}\right)
$$

where $\sigma_{0}$ is the yield stress. Here, the plastic strain and strain rate are denoted by $\epsilon_{0}$ and $\dot{\epsilon}_{0}$, respectively, to indicate that Eq. (1) refers to quantities varying instantaneously (adiabatic approximation, cf. Eq. (3)). Note that $f\left(\dot{\epsilon}_{0}\right)$ is $\mathrm{N}$-shaped and, hence, contains the essential nonlinearity of the problem.

Eq. (1) may be used to describe singular (i.e. discontinuous) relaxation oscillations of $\dot{\epsilon}_{0}$. It is, however, inadequate in (i) specifying the propagation velocity of deformation bands and (ii) accounting for the intermittent propagation of type B bands observed in strain-rate control. Two modifications have been suggested to eliminate these shortcomings.

(i) According to a proposal by Aifantis [6], internal stresses $\sigma_{\text {int }}$ which provide some kind of spatial coupling have to be included when $\sigma_{f}$ is related to the external tensile stress $\sigma_{\text {ext }}$ :

$$
\sigma_{\text {ext }}=\sigma_{\mathrm{f}}-\sigma_{\mathrm{int}}, \quad \sigma_{\mathrm{int}}=-C \partial_{x}^{2} \epsilon_{0} \quad \text { with } \quad C>0
$$

Here, $x$ stands for the coordinate along the tensile direction. In fact, Eq. (2) represents the simplest modification possible, as first order gradients are forbidden for symmetry reasons (inversion symmetry) and $C<0$ does not allow for a local strain rate maximum corresponding to a deformation band. In order to assess the physical origin of the coefficient $C$, various coupling mechanisms (due to crossslip, non-local hardening and incompatibility stresses) have been compared in [7]. It has been shown that incompatibility stresses prevail in polycrystalline samples (used in most experiments). In the case of a completely elastic accomodation of incompatibilities of plastic strain, $C$ can be estimated to be $C \approx G d^{2} / 4$ where $G$ and $d$ are the shear modulus and the grain size, respectively. It is important to note that the rôle of the internal stresses is similar to that played by electron diffusion in the Gunn effect, as, in both cases, gradient terms are required to remove singularities and to determine the propagation velocity ( $c f$. Table 1 ). 
(ii) In a DSA alloy, dislocation dynamics exhibits a finite response time, since any change of the deformation path is accompanied by diffusional rearrangements of the solute atmospheres and a redistribution of the ageing times of dislocations. Eq. (1) is not appropriate to describe rapid changes of the deformation process, since the DSA time scale is neglected, corresponding to an adiabatic elimination of the dynamics of the solute concentration $C_{\mathrm{s}}$ in the dislocation cores [8]. Assuming an exponential ageing kinetics at a rate $\nu$ and performing a statistical analysis of the coupled dynamics of dislocations and solute atoms, the true strain rate $\dot{\epsilon}$ can be shown to obey a relaxator behaviour [9]:

$$
\partial_{t} \dot{\epsilon}=-\frac{\dot{\epsilon}-\dot{\epsilon}_{0}}{\tau} \quad \text { with } \quad \tau=\nu \frac{\delta G}{k_{\mathrm{B}} T} \frac{\dot{\epsilon}_{0} / \delta \epsilon}{\left(\dot{\epsilon}_{0} / \delta \epsilon+\nu\right)^{3}} .
$$

Here, $k_{\mathrm{B}}$ is Boltzmann's constant, $\delta G$ is the total increase due to DSA of the Gibbs' enthalpy of thermal dislocation activation and $\delta \epsilon$ is the elementary strain increment per activation step. Note that there is no retardation $\left(\tau=0, \dot{\epsilon}=\dot{\epsilon}_{0}\right.$ ) both for $\dot{\epsilon}_{0}=0$ (complete ageing) and $\dot{\epsilon}_{0} \rightarrow \infty$ (effectively no ageing), while the memory effect is maximum for $\dot{\epsilon}_{0}=\nu \delta \epsilon / 2$, i.e. for strain rates of the order of magnitude of the ageing rate.

Combining Eqs. (1-3) and retaining only the first order of $\tau, \epsilon_{0}$ may be eliminated yielding

$$
\partial_{t} \sigma_{\text {ext }}=\left(1+\tau \partial_{t}\right)\left(C \partial_{x}^{2} \dot{\epsilon}+g(\dot{\epsilon}) \partial_{t} \dot{\epsilon}+h \dot{\epsilon}\right) \quad \text { with } \quad g=\partial_{\dot{\epsilon}} f .
$$

For any value of $\partial_{t} \sigma_{\text {ext }}$, Eq. (4) exhibits a pulse-like solution that travels as a solitary wave along the gauge length $l(0<x<l)$ and that satisfies the clamping conditions $\dot{\epsilon}(x=0)=\dot{\epsilon}(l)=0$. Practically, this means that the propagation velocity $c$ is determined in a unique way for constant stress-rate testing. It is interesting to note that periodic travelling wave trains that Eq. (4) allows for as well, may propagate at arbitrary $c$. This illustrates that the velocity selection depends on the type of boundary conditions imposed. Using a piecewise linear caricature of the $f(\dot{\epsilon})$ characteristic yields the propagation velocity [7]

$$
c=\frac{\left[G \dot{\epsilon}_{2}\left(h \dot{\epsilon}_{2}-\partial_{t} \sigma_{\mathrm{ext}}\right)\right]^{1 / 2} d}{f_{2}},
$$

where $f_{2}=f\left(\dot{\epsilon}_{2}\right)$ and $\dot{\epsilon}_{2}$ denote the friction stress and the strain rate at the minimum of the characteristic.

Furthermore, Eq. (4) may be combined with the machine condition,

$$
\dot{L}=\int_{0}^{l} \mathrm{~d} x \dot{\epsilon}+M_{\mathrm{eff}} l \partial_{t} \sigma_{\mathrm{ext}}
$$

( $\dot{L}$ and $M_{\text {eff }}$ denote the cross-head velocity and the effective compliance of the tensile system, respectively) in order to investigate solutions for $\dot{L}=$ const. testing. The results obtained cover solitary waves (type A bands occurring at large $\dot{L}$ ), intermittently propagating waves (type B bands at intermediate $\dot{L}$ ) and non-propagative solutions (type $\mathrm{C}$ bands at small $\dot{L}$ ), as well as the transition conditions between these different modes [7]. As the agreement with experimental findings is quite well, Eqs. $(4,6)$ are supposed to consitute a complete model for the variety of deformation modes classed under the term "PLC effect".

\section{Lüders Bands}

Whereas the PLC effect denotes the repeated yielding due to a type $S$ instability, a Lüders band (LB) is a dislocation avalanche moving once into unyielded material. It is accomplished by softening owing to rapid dislocation multiplication during the propagation of a deformation band (type $h$ instability [1]). The way of modelling must, of course, reflect this important difference. As demonstrated in the preceding section, the PLC effect is conveniently described in terms of the local plastic strain rate, since the phenomenon is governed by variations of the dislocation glide velocity $v$ while the spatio-temporal evolution of dislocation densities is negligible. Quite the reverse is true for a LB. Here, the coupled dynamics of different dislocation species is important while $v$ plays a minor role. Again the question in the focus of interest is concerning the mechanisms that the deformation band is propagated by. 


\subsection{Propagation Mechanisms}

"Classical" LB propagation, known to metallurgists for a long time, is observed in low-ductility bcc polycrystals, in particular mild steel. A dislocation dynamical model must reflect the polycrystalline peculiarities of yielding. Consider the dynamics of mobile dislocations in terms of the scalar density $\rho_{\mathrm{m}}(x)$, as a function of the coordinate $x$ perpendicular to the LB front. As illustrated in Fig. 2,

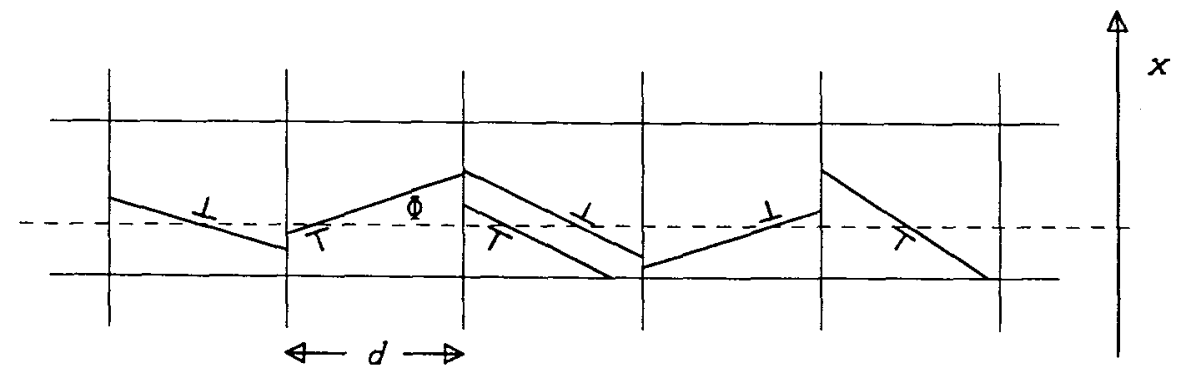

Figure 2: Diffusion-like dislocation dynamics due to the random crystallographic grain orientation.

$\rho_{\mathrm{m}}$ comprises dislocations belonging to various grains with different active slip systems of random crystallographic orientation. Consequently, the dynamics of $\rho_{\mathrm{m}}$ is stochastic with respect to the $x$ axis. On an average performed over a specimen cross-section $(x=$ const.) containing many grains, this may be expressed by [10]

$$
\partial_{t} \rho_{\mathrm{m}}=\Delta \partial_{x}^{2} \dot{\gamma}+\dot{\rho}_{\mathrm{m}}^{(+)}-\dot{\rho}_{\mathrm{m}}^{(-)} \quad \text { with } \quad \Delta=\Delta_{\text {poly }}=\left(\frac{\delta m}{2}\right)^{2} \frac{d}{b} .
$$

Here, $b$ is the Burgers vector, $\delta m$ is the mean variation of the orientation factor, and $\dot{\gamma}=b \rho_{\mathbf{m}} v$ denotes the average resolved shear strain rate. Before specifying the rates of dislocation generation, $\dot{\rho}_{m}^{(+)}$, and dislocation storage, $\dot{p}_{\mathrm{m}}^{(-)}$, mechanisms of LB propagation in single crystals are presented.

LB (sometimes referred to as Lüders-like slip) are also observed in single crystals, namely solution or irradiation-hardened fcc crystals [11] and semiconductor crystals [12] oriented for single glide. A remarkable peculiarity is the hierarchical slip structure found in neutron irradiated $\mathrm{Cu}[11,13]$. It consists of slip lines clustered in slip bands which, in turn, are clustered in slip band bundles. The LB consisting of several active slip band bundles propagates by successively forming another bundle in the front. There is experimental evidence that, within the slip bands and slip band bundles, slip transfer occurs by cross-slip. On the one hand, cross-slip constitutes an important length scale (crossslip height $h_{c s}$ ), on the other hand it contributes to dislocation multiplication, as a cross-slipped screw
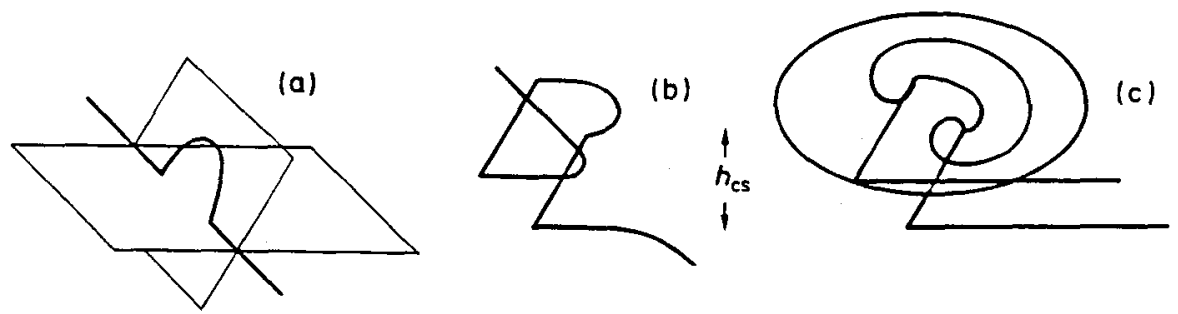

Figure 3: Slip propagation and dislocation multiplication by double cross-slip; cross-slip height $h_{\mathrm{cs}}$.

dislocation segment may expand on an adjacent primary slip plane (Fig. 3) and form a Frank-Read source. These two processes may be cast into the form of Eq. (7) with

$$
\Delta=\Delta_{\text {single }}=\frac{\pi}{2} q_{\mathrm{FR}} \frac{\tau_{\mathrm{eff}} h_{\mathrm{cs}}^{2}}{G b^{2}} \quad \text { and } \quad \dot{\rho}_{\mathrm{m}}^{(+)}=\pi q_{\mathrm{FR}} \frac{\tau_{\mathrm{eff}} \dot{\gamma}}{G b^{2}}
$$


Here the dimensionless fraction $q_{\mathrm{FR}} \approx 10^{-1}$ of cross-slip events leading to the formation of Frank-Read sources and the effective resolved shear stress $\tau_{\text {eff }}$ have been introduced [14].

The macroscopic LB propagation cannot, however, be attributed to cross-slip, because the separation of slip band bundles exceeds clearly the largest cross-slip heights observed. It has been suggested that the nucleation of a fresh bundle in the LB front is brought about by non-axial stresses caused by the non-uniformity of deformation [11]. In fact, this is supported by the experimental finding that the bundle distance increases in proportion to the thickness $s$ of the specimen and that slip band clustering gives way to a disperse distribution of slip bands if $s<200 \mu \mathrm{m}$. In thin flat specimens used by Neuhäuser et al. [11,13], a biaxial stress state develops in the LB front (Fig. 4). The corresponding average resolved shear stress within a primary slip plane $(x=$ const.) may be expressed in terms of the curvature of the distribution of shear $\gamma(x)$;

$$
\text { eff }=\tau_{\text {ext }}-\tau_{0}+\frac{\tau_{\text {ext }}}{8} s^{2} \partial_{x}^{2} \gamma,
$$

where $\tau_{\text {ext }}$ and $\tau_{0}$ denote the resolved external stress and the critical resolved shear stress, respectively [14].

The spatial couplings involved in Eqs. (7), (8) and (9) constitute different propagation mechanisms based on intrinsic (grain size $d$, cross-slip height $h_{c s}$ ) or extrinsic (specimen thickness $s$ ) length scales. Each of those expressions is obtained from Taylor expansion retaining only the first non-zero

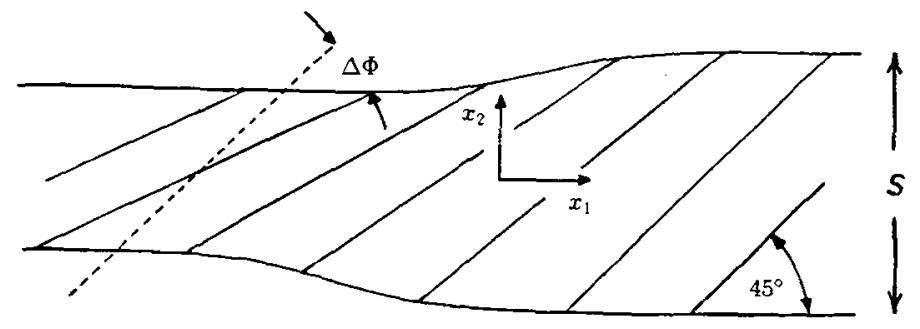

Figure 4: Sketch of the LB front in a tensile specimen showing the non-uniform distribution of slip and the corresponding lattice rotation. This gives rise to a biaxial stress state.

term (actually, the second order derivative for symmetry reasons). In what follows, we shall outline how to incorporate the propagation mechanisms into dislocation dynamical models, both for the polycrystalline (Sect. 3.2) and the single crystalline case (Sect. 3.3), and which results derive.

\subsection{Lüders Bands in Polycrystals}

It is well-known that the lower yield stress (propagation stress $\sigma_{\text {prop }}$ ) of bcc metals obeys the HallPetch law $\left(\sigma_{\text {prop }} \sim d^{-1 / 2}\right)$. This is commonly explained by assuming that the intergranular slip transfer is caused by short-range stress concentrations due to dislocation pile-ups. To account for this we consider the density $\rho_{\mathrm{p}}$ of piled-up dislocations as a dynamical variable. Furthermore, strain hardening due to dislocation storage within the LB is described in terms of the density $\rho_{\mathrm{i}}$ of immobile dislocations. Slip propagation by cross-slip may be neglected as $\Delta_{\text {poly }} \gg \Delta_{\text {single. }}$. The coupled dynamics of $\left\{\rho_{\mathrm{m}}, \rho_{\mathrm{p}}, \rho_{\mathrm{i}}\right\}$ reads $[10]$ :

$$
\begin{aligned}
\partial_{t} \rho_{\mathrm{m}} & =\Delta_{\mathrm{poly}} \partial_{x}^{2} \dot{\gamma}+\mathcal{Q}+\frac{d \tau^{*}}{\alpha G b \lambda} \Theta\left(\rho_{\mathrm{p}}-\rho_{\mathrm{p} 0}\right) v \rho_{\mathrm{p}}-\frac{v}{d} \rho_{\mathrm{m}}-\beta \sqrt{\rho_{\mathrm{t}}} v \rho_{\mathrm{m}} \\
\partial_{t} \rho_{\mathrm{p}} & =\frac{v}{d} \rho_{\mathrm{m}}-\frac{v}{\lambda} \rho_{\mathrm{p}} \\
\partial_{t} \rho_{\mathrm{i}} & =\beta \sqrt{\rho_{\mathrm{t}}} v \rho_{\mathrm{m}}+\frac{v}{\lambda} \rho_{\mathrm{p}}
\end{aligned}
$$

with the total dislocation density $\rho_{\mathrm{t}}=\rho_{\mathrm{m}}+\rho_{\mathrm{p}}+\rho_{\mathrm{i}} . \mathcal{Q}$ standing for the dislocation generation due to grown-in sources is a measure of the ductility of the polycrystal. The third term on the r.h.s. of Eq. (10) describes dislocation multiplication mediated by pile-ups (dynamical Hall-Petch mechanism), the 
rate of which is governed by the effective resolved stress $\tau^{*}$ acting on grain boundaries and the range $\lambda$ of stress concentrations. As this process requires at least one pile-up per grain $\left(\rho_{\mathrm{p}}>\rho_{\mathrm{p} 0}=n d^{-2}\right.$; $n$ is the number of dislocations in a pile-up), the Heaviside step function $\Theta$ is introduced. $\alpha$ and $\beta$ are numerical factors of the order of unity and $10^{-2}$, respectively. The last two terms of Eq. (10) stand for dislocation storage at grain boundaries and in the grain interiors, resulting in corresponding production terms of piled-up and immobile dislocations, respectively. Finally, the last terms of Eqs. (11) and (12), respectively, express the plastic relaxation of pile-ups.

Approximate solutions of Eqs. (10-12) corresponding to the nucleation and propagation of a LB may be found using time scale separation techniques. The results are in good accordance with experiments and may be summarized as follows [10]: (i) The upper yield stress (nucleation stress) is in inverse proportion to the width of the nucleus and decreases with increasing $\mathcal{Q}$. If the ductility exceeds a critcal value the LB phenomenon gives way to uniform yielding. (ii) LB propagation corresponds to a solitary wave in a bistable excitable medium. While it is propagating, the metastable ground state of low $\rho_{\mathrm{m}}$ is replaced by the stable excited state of large $\rho_{\mathrm{m}}$. The propagation velocity is determined in a unique way by the lower yield stress and is proportional to the glide velocity $v$. (iii) The Lüders strain decreases with increasing grain size as $d^{-5 / 8}$, and it increases as the logarithm of the imposed cross-head velocity. The width of the LB is in proportion to $d^{5 / 8}$.

\subsection{Lüders Bands in Single Crystals}

Here, dislocation dynamics corresponds to stage I of the stress-strain curve, i.e., strain hardening within the LB is due to the formation of dislocation dipoles (which may annihilate spontaneously in the case of screw dislocations). This gives rise to a term $\dot{\rho}_{\mathrm{m}}^{(-)} \sim \rho_{\mathrm{m}}^{2}$ and from Eqs. (7-9) we get [14]

$$
\begin{aligned}
\partial_{t} \rho_{\mathrm{m}} & =\Delta_{\text {single }} \partial_{x}^{2} \dot{\gamma}+\frac{\pi q_{\mathrm{FR}}}{G b}\left(\tau_{\mathrm{ext}}-\tau_{0}+\frac{\tau_{\mathrm{ext}}}{8} s^{2} \partial_{x}^{2} \gamma\right) v \rho_{\mathrm{m}}-\frac{G b}{2 \pi\left(\tau_{\text {ext }}-\tau_{0}\right)} v \rho_{\mathrm{m}}^{2}, \\
\partial_{t} \gamma & =b v \rho_{\mathrm{m}} .
\end{aligned}
$$

Again, LB propagation is associated with solitary wave solutions linking the excited state, $\rho_{\mathbf{m}}=$ $2 q_{\text {FR }}\left[\pi\left(\tau_{\text {ext }}-\tau_{0}\right) / G b\right]^{2}$, with the ground state, $\rho_{\mathrm{m}}=0$. As an important difference to the polycrystalline case, the ground state is unstable now, as revealed by LSA. This is why a continuous family of solitary wave solutions exists that are parametrized by different values of the propagation velocity $c$. Hence, the question of propagation velocity selection leading to some asymptotic value of $c$ is to be addressed. This is achieved by analyzing the phase dynamics [15] within the leading and the trailing edges of the solitary waves. It turns out that two attractors of $c$ corresponding to different modes of propagation exist [14]. (i) If LB propagation is mainly due to cross-slip a convectively stable solitary wave develops asymptotically, propagating at a velocity

$$
c=2 \sqrt{2} \pi q_{\mathrm{FR}} \frac{\left(\tau_{\mathrm{ext}}-\tau_{0}\right) h_{\mathrm{cs}}}{G b} v .
$$

Clustering of slip bands does not occur. (ii) If, however, the propagation is perturbed by biaxial stresses, stabilization of the wave is prevented. In this case, numerical integration of Eqs. $(13,14)$ has revealed that the LB is propagated in an intermittent manner [14] quite similar to the successive formation of fresh slip band bundles found in experiment $[11,13]$. The critical specimen thickness for the transition to slip band clustering, as derived from theory, $s>s_{\text {crit }}=2\left(2 G / \pi \tau_{\text {ext }}\right)^{1 / 2} h_{\text {cs }}$, agrees well with experimental findings.

\section{Thermomechanical Fronts}

During low-temperature plastic deformation of single- as well as of polycrystals, repeated instabilities may develop. Similar to the PLC-Effect, these instabilities lead to staircase-like stress-strain curves during stress-rate controlled tests, and different types of serrated stress-strain curves during strain-rate control [16]. They result from a positive feedback between heat generation during plastic deformation and acceleration of dislocations due to a rise of temperature [17] (type $T$ instability [1]). Thus, the temperature $T$ is an additional dynamical variable, i.e., the equations describing the dislocation 
dynamics have to be complemented by the equation of heat conduction. Assuming a specimen with large aspect ratio $l / r$ ( $l$ is the specimen length and $r$ the specimen radius) it reads

$$
c_{\mathrm{V}}^{0} T^{3} \frac{\partial T}{\partial t}=\kappa \frac{\partial^{2} T}{\partial x^{2}}-\frac{k}{2 r}\left(T-T_{\mathrm{ext}}\right)+\tau_{\mathrm{ext}} \dot{\gamma}
$$

Here $c_{\mathrm{V}}^{0} T^{3}$ is the specific heat of the material, $\kappa$ is the heat conductivity and $k$ is the coefficient of heat transfer through the surface of the specimen embedded into a coolant kept at fixed temperature $T_{\text {ext }}$. The average resolved shear strain rate $\dot{\gamma}$ increases strongly with $T$ through the $T$-dependence of the dislocation glide velocity,

$$
v\left(\tau_{\text {eff }}, T\right)=v_{0} \exp \left(-\frac{H\left(\tau_{\text {eff }}\right)}{k_{\mathrm{B}} T}\right) .
$$

Here, $v_{0}$ is a preexponential factor of the order of magnitude of the velocity of sound, and $H$ is the effective activation enthalpy which depends on the effective resolved shear stress $\tau_{\text {eff }}=\tau_{\text {ext }}-\tau_{0}-$ $\alpha G b \sqrt{\rho_{\mathrm{t}}}$.

After a certain amount of predeformation, a large dislocation density is accumulated. As storage is mainly due to dislocation reactions, the dynamics may be written in the following simplified form, which applies for both polycrystals and single crystals deforming in a multislip mode [18]:

$$
\begin{aligned}
\partial_{t} \rho_{\mathrm{m}} & =\frac{\pi}{G b}\left(\tau_{\mathrm{eff}}-\tau_{\mathrm{c}}\right) \Theta\left(\tau_{\mathrm{eff}}-\tau_{\mathrm{c}}\right) \rho_{\mathrm{m}} v-\beta \sqrt{\rho_{\mathrm{t}}} \rho_{\mathrm{m}} v, \\
\partial_{t} \rho_{\mathrm{i}} & =\beta \sqrt{\rho_{\mathrm{t}}} \rho_{\mathrm{m}} v .
\end{aligned}
$$

Compared to Eqs. (10-12) all influences of grain boundaries are neglected. By contrast, the first term on the r.h.s of Eq. (18) describes intragranular dislocation multiplication. $\tau_{c}$ is the resolved stress required to operate a dislocation source.

Eqs. (16-19) are coupled through the $T$-dependence of the dislocation glide velocity. Therefore, the spatial coupling arising from heat conduction also influences the dislocation dynamics. Since, at low temperatures, this coupling mechanism is much more efficient than those discussed in the previous sections, other gradient terms need not be taken into account.

Eqs. (16-19) exhibit a solitary wave solution ('thermomechanical front') that satisfies the boundary conditions $T(x=0)=T(x=l)=T_{\text {ext }}, \rho_{\mathrm{m}}(x=0)=\rho_{\mathrm{m}}(x=l)=0$. The structure of this wave-type solution is illustrated in Fig.5. Because of the strong (exponential) nonlinearity of the heat production term, almost all glide activity and heat production are localized within a narrow active region where the temperature is close to its maximum value $T_{\mathbf{m}}[18]$. The amount of heat release is limited by the

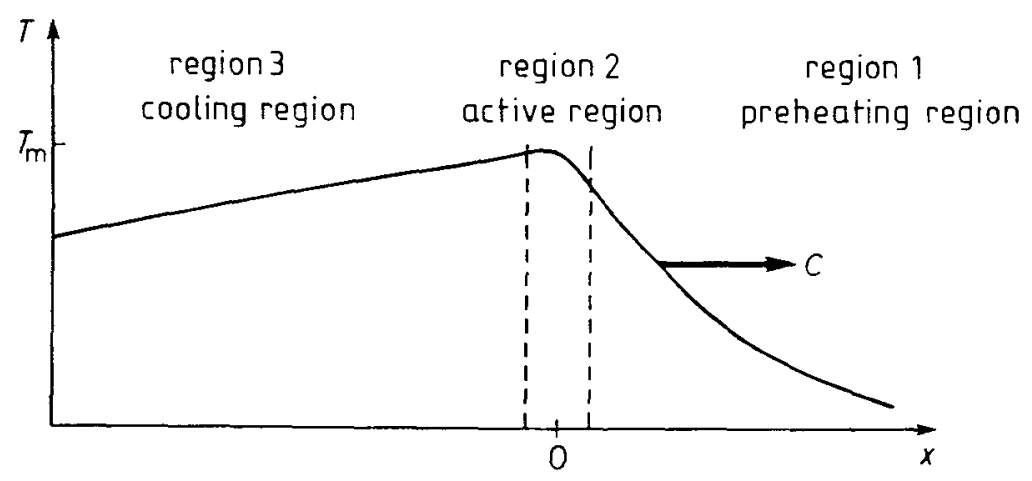

Figure 5: Schematic representation of the temperature profile of a thermomechanical front. (1) preheating region, (2) active region, (3) cooling region. 
relaxation of mobile dislocations into immobile configurations at the rear of this region, leaving the specimen in a strongly hardened state. The active region is followed by a cooling region where the specimen temperature drops, due to heat losses through the surfaces, down to its initial value $T_{\text {ext }}$.

Ahead of the active region there is a preheating region. There heat conduction from the active region leads to a rise of temperature from its asymptotic value to the maximum temperature. While heat production is still negligible, dislocation multiplication already sets in such that the mobile dislocation density reaches its maximum value at the front edge of the active region.

The active region forms a boundary layer between the two outer regions. Its structure may be studied by the method of matched asymptotic expansions [19]. As a result of the matching conditions between the active and the outer regions, the propagation velocity is determined in a unique way:

$$
c=\bar{c}\left\{\frac{4 \kappa k_{\mathrm{B}}}{c_{\mathrm{V}} \sqrt{T_{\mathrm{m}}^{4}-T_{\mathrm{ext}}^{4}} H\left(\tau_{\mathrm{c}}\right)} \beta \sqrt{\rho_{\mathrm{t}}} v\left(T_{\mathrm{m}}, \tau_{\mathrm{c}}\right)\right\}^{1 / 2} .
$$

Here, $\bar{c}$ is a dimensionless prefactor of the order of unity.

In order to investigate solutions for $\dot{L}=$ const. testing, Eqs. (16) to (18) are combined with the machine equation (6). Solitary waves similar to the type A bands of the PLC effect as well as nonpropagative solutions analogous to the type $\mathrm{C}$ bands are found, and transition conditions between these different modes can be established [18]. In view of the fact that almost no experimental data on the spatio-temporal behaviour of low-temperature plastic instabilities are available, an experimental check of the model predictions would be highly desirable and may provide a key problem of future experimental work in the field of unstable plastic flow at low temperatures.

\section{Conclusions}

The paper gives a survey of the physical mechanisms of coherently propagating modes of plastic deformation and, in particular, solitary plastic waves. The examples discussed cover the Portevin-le Châtelier effect, Lüders bands and thermomechanical fronts. In each case, the dynamics of defects

Table 2: Synopsis of propagative deformation modes.

\begin{tabular}{|c|c|c|c|c|}
\hline & $\overline{\text { PLC Effect }}$ & $\begin{array}{c}\text { Lüders Bands in } \\
\text { Polycrystals }\end{array}$ & $\begin{array}{l}\text { Lüders Bands in } \\
\text { Single Crystals }\end{array}$ & $\begin{array}{c}\text { Thermomechanical } \\
\text { Fronts }\end{array}$ \\
\hline $\begin{array}{c}\text { Plastic } \\
\text { Instability }\end{array}$ & type $S$ & $\overline{\text { type } h}$ & type $h$ & type $\bar{T}$ \\
\hline $\begin{array}{l}\text { Dynamical } \\
\text { Variables }\end{array}$ & $\overline{C_{\mathrm{s}}}, \dot{\boldsymbol{\epsilon}}$ & $\rho_{\mathrm{m}}, \rho_{\mathrm{p}}, \rho_{\mathrm{i}}$ & $\rho_{\mathrm{m}}, \gamma$ & $T, \rho_{\mathrm{m}}, \rho_{\mathrm{i}}$ \\
\hline $\begin{array}{l}\text { Length Scales } \\
\text { (Propagation } \\
\text { mechanisms) }\end{array}$ & $\begin{array}{c}\text { grain size } d \\
\text { (incompatibility } \\
\text { stresses) }\end{array}$ & $\begin{array}{l}\text { grain size } \bar{d} \\
\text { (random grain } \\
\text { orientation) }\end{array}$ & $\begin{array}{c}\text { cross-slip } h_{\mathrm{cs}}, \\
\text { specimen thickness } s \\
\text { (cross-slip, } \\
\text { biaxial stresses) }\end{array}$ & heat conduction \\
\hline Time Scales & $\begin{array}{c}\text { DSA rate, } \\
\text { strain hardening }\end{array}$ & $\begin{array}{l}\text { strain hardening, } \\
\text { dislocation } \\
\text { multiplication, } \\
\text { plastic relaxation } \\
\text { of pile-ups }\end{array}$ & $\begin{array}{l}\text { strain hardening, } \\
\text { dislocation } \\
\text { multiplication }\end{array}$ & $\begin{array}{l}\text { strain hardening, } \\
\text { dislocation } \\
\text { multiplication }\end{array}$ \\
\hline Nonlinearities & $f(\dot{\epsilon})$ & $\begin{array}{c}\text { dislocation } \\
\text { multiplication }\end{array}$ & $\begin{array}{c}\text { dipole formation, } \\
\text { biaxial stresses }\end{array}$ & $\begin{array}{c}T \text {-dependence of } \\
\text { dislocation velocity }\end{array}$ \\
\hline $\begin{array}{c}\text { Initial } \\
\text { Conditions }\end{array}$ & & nucleus width & nucleus width & \\
\hline $\begin{array}{l}\text { Boundary } \\
\text { Conditions }\end{array}$ & $\dot{\dot{\epsilon}}(0)=\dot{\epsilon}(\bar{l})=0$ & & & $\begin{array}{l}T(0)=T(l)=T_{\text {ext }} \\
\rho_{\mathrm{m}}(0)=\rho_{\mathrm{m}}(l)=0\end{array}$ \\
\hline
\end{tabular}


(dislocations) provides a clue to understanding what length and time scales govern the plastic-wave propagation and what kind of nonlinearities cause the localization of plastic deformation. This is summarized in Table 2. As to the Lüders bands, the appearance of a plastic wave depends on the width of the nucleus and, thus, on the initial conditions. Moreover, the propagation velocity, as derived from theory, may depend sensitively on the type of boundary conditions considered, as is the case for the PLC effect and thermomechanical fronts.

\section{Acknowledgements}

Stimulating discussions with W. Frank, L.P. Kubin and H. Neuhäuser are gratefully acknowledged.

\section{References}

[1] Y. Estrin and L.P. Kubin, Res Mechanica 23 (1988) 197.

[2] Y. Estrin and L.P. Kubin, J. Mech. Beh. Met. 2 (1990) 255.

[3] L.P. Kubin and Y. Estrin, J. Physique 47 (1986) 497.

[4] B.W. Knight and G.A. Peterson, Phys. Rev. 155 (1967) 393.

[5] P. Penning, Acta metall. 20 (1972) 1169.

[6] H.M. Zbib and E.C. Aifantis, Scripta metall. 22 (1988) 1331.

[7] P. Hähner, Mat. Sci. Eng. A, in the press.

[8] P.G. McCormick, Acta metall. 36 (1988) 3061.

[9] P. Hähner, to be published.

[10] P. Hähner and L.P. Kubin, Solid State Phenomena 23\&24 (1992) 385.

[11] H. Neuhäuser, in: Dislocations in Solids 6, F.R.N. Nabarro (ed.), North-Holland, Amsterdam (1983), p.319.

[12] H. Alexander, in: Dislocations in Solids 7, F.R.N. Nabarro (ed.), North-Holland, Amsterdam (1986), p.113.

[13] H. Neuhäuser, Solid State Phenomena $3 \& 4$ (1988) 407.

[14] P. Hähner, Versetzungsdynamik propagierender plastischer Verformungsmoden, Thesis, Univ. Stuttgart (1992); P. Hähner, submitted to Appl. Phys.

[15] W. van Saarloos, Phys. Rev. A 37 (1988) 211.

[16] T. Ogata, K. Ishikawa, O. Umezawa, and T. Yuri, Cryogenics 28 (1988) 511; Adv. Cryog. Eng. 34 (1988) 209.

[17] Y. Estrin and L.P. Kubin, in: Continuum Models of Discrete Systems, O. Brulin and R. K. T. Hsieh (eds.), North Holland, Amsterdam (1981), p. 13

[18] M. Zaiser, Appl. Phys., in the press.

[19] G. Shivashinsky, J. Chem. Phys. 62 (1975) 638. 